\title{
DESEMPENHO DE ESCOLARES COM DISLEXIA E TRANSTORNO DO DÉFICIT DE ATENÇÃO E HIPERATIVIDADE NOS PROCESSOS DE LEITURA
}

\section{PERFORMANCE OF STUDENTS WITH DYSLEXIA AND ATTENTION DEFICIT DISORDER WITH HYPERACTIVITY IN THE READING ASSESSMENT PROCESSES}

\author{
Adriana Marques de Oliveira ${ }^{1}$ \\ Monique Herrera Cardoso ${ }^{2}$ \\ Fábio Henrique Pinheiro ${ }^{3}$ \\ Giseli Donadon Germano ${ }^{4}$ \\ Simone Aparecida Capellini ${ }^{5}$
}

Oliveira AM et al. Desempenho de escolares com dislexia e transtorno do déficit de atenção e hiperatividade nos processos de leitura. Rev Bras Cresc e Desenv Hum 2011; 21(2): 344-355.

\section{RESUMO}

Este estudo tem por objetivos caracterizar e comparar o desempenho de escolares com diagnóstico interdisciplinar de dislexia e TDAH com escolares com bom desempenho acadêmico nos processos de leitura. Participaram 60 escolares de ambos os gêneros, de $2^{\mathrm{a}}$ a $4^{\mathrm{a}}$ série do ensino fundamental, divididos da seguinte forma: GI, 20 escolares com dislexia; GII, 20 escolares com TDAH, e GIII, 20 escolares com bom desempenho acadêmico, pareados segundo gênero, faixa etária e escolaridade com o GI e o GII. Os escolares foram submetidos à aplicação das provas de Avaliação dos Processos de Leitura - PROLEC, composta pelos processos de identificação de letras, léxico, sintático e semântico. Os resultados evidenciaram que os escolares do GIII apresentaram desempenho superior em relação ao GI e ao GII. Os escolares do GI e do GII apresentaram diferença estatisticamente significante apenas nas provas de leitura de palavras não frequentes e leitura de não palavras do processo léxico. A dificuldade apresentada pelo GI e pelo GII nas provas do PROLEC pode ser justificada pela dificuldade de codificação e decodificação de símbolos. Nos escolares com TDAH, essa alteração se deve ao comprometimento da interação entre o processamento visual, linguístico, atencional e auditivo, e nos escolares com dislexia é em decorrência de falha no processo de mediação fonológica, que depende da utilização do conhecimento das regras de conversão grafema-fonema para a construção da leitura da palavra. Essas alterações comprometem a realização da leitura e a compreensão do material lido.

Palavras-chave: leitura; dislexia; transtorno do déficit de atenção com hiperatividade.

1 Fonoaudióloga, Mestranda do Programa de Pós Graduação em Educação da Faculdade de Filosofia e Ciências - FFC - UNESP - Marília (SP), Brasil. Avenida Hygino Muzzy Filho, 737, Campus Universitário, CEP: 17-525-900. Bolsista do Conselho Nacional de Desenvolvimento Científico e Tecnológico - CNPq.E-mail: adrimaroli@yahoo.com.br

2 Fonoaudióloga do Laboratório de Investigação dos Desvios da Aprendizagem do Departamento de Fonoaudiologia da Faculdade de Filosofia e Ciências - FFC - UNESP - Marília (SP), Brasil. Avenida Hygino Muzzy Filho, 737, Campus Universitário, CEP: 17-525-900. E-mail: moniquehc@gmail.com

3 Fonoaudiólogo. Mestre em Educação. Doutorando do Programa de Pós Graduação em Educação da Faculdade de Filosofia e Ciências - FFC - UNESP - Marília (SP), Brasil. Avenida Hygino Muzzy Filho, 737, Campus Universitário, CEP: 17-525-900. Bolsista da Coordenação de Aperfeiçoamento de Pessoal de Nível Superior-CAPES. E-mail: fabiohenriquepinheiro@yahoo.com.br

4 Fonoaudióloga. Doutora em Educação pela Faculdade de Filosofia e Ciências, FFC - UNESP - Marília(SP), Brasil. E-mail: giseliger@yahoo.com.br

5 Fonoaudióloga. Livre-docente em Linguagem Escrita. Docente do Departamento de Fonoaudiologia e Programa de Pós-Graduação em Educação da Faculdade de Filosofia e Ciências da Universidade Estadual Paulista - FFC - UNESP - Marilia (SP), Brasil. Avenida Hygino Muzzy Filho, 737, Campus Universitário, CEP: 17.525-900. E-mail: sacap@uol.com.br

Trabalho realizado no Laboratório de Investigação dos Desvios da Aprendizagem do Departamento de Fonoaudiologia da Faculdade de Filosofia e Ciências da Universidade Estadual Paulista - LIDA- FFC - UNESP - Marilia (SP), Brasil.

Correspondência para: Simone Aparecida Capellini. E-mail: sacap@uol.com.br 


\begin{abstract}
This study aimed to characterize and to compare the performance of students with an interdisciplinary diagnosis of dyslexia and attention deficit hyperactivity disorder (ADHD) in students with good academic performance on the reading processes. Sixty students from both genders, from $2^{\text {nd }}$ to $4^{\text {th }}$ grades of municipal public schools in Marília - SP participated in this study, they were distributed as follows: GI, 20 students with interdisciplinary diagnosis of dyslexia; GII, 20 students with ADHD and GIII, 20 students with good academic performance, paired according to gender, age and grade level with GI and GII. The students were submitted to the application of the assessment of reading processes (PROLEC) composed by four processes: letters identification, lexical, syntactical and semantic. The results highlighted that the students of GIII showed superior performance comparing with GI and GII. There was difference between GI and GII only in low frequency word reading and non words reading of the lexical process. The inferior performance from GI and GII in the PROLEC tests can be justified by the difficulty on the coding and decoding abilities. In ADHD students this difficulty was due to impaired interaction between the visual, linguistic, attention and auditory processing and in the dyslexic students was due to failure at the phonological mediation process, which depends on the knowledge of rules of grapheme - phoneme conversion to the acquisition of word reading. These changes affect the reading achievement and the comprehension of the read text.
\end{abstract}

Key words: reading; dyslexia; attention deficit disorder with hyperactivity.

\section{IINTRODUÇÃO}

O desenvolvimento da leitura requer a organização multidimensional e dinâmica de várias habilidades. A relação entre linguagem, atenção, identificação de palavras, vocabulário, compreensão, experiência, fonologia, inteligência, instrução e fluência tem, assim como para outras habilidades, demonstrado ter forte impacto no desenvolvimento da leitura ${ }^{1}$.

Uma das habilidades mais importantes para o aprendizado da leitura é a consciência fonológica, a qual se desenvolve à medida que a criança é submetida a complexas tarefas linguísticas, permitindo que identifique e manipule as unidades, silábicas ou fonêmicas, de uma palavra ${ }^{2}$, de forma a compreender, assim, o princípio alfabético da correspondência grafema-fonema ${ }^{3,4}$.

Para o desenvolvimento da consciência fonológica, é necessária uma relação entre memória de trabalho e desempenho em atividades $^{4}$, portanto escolares que apresentem um déficit nessa relação podem vir a apresentar dificuldades no aprendizado da leitura. Exemplos de escolares com esse déficit são crianças com transtorno do déficit de atenção e hiperatividade (TDAH) e crianças disléxicas.
O transtorno de déficit de atenção e hiperatividade é o distúrbio neuropsiquiátrico mais comum da infância e está incluído entre as doenças crônicas mais prevalentes entre escolares, podendo persistir até a idade adulta ${ }^{5-8}$. São características do quadro: a desatenção, a hiperatividade e a impulsividade, que podem variar em maior ou menor grau ${ }^{9-11}$, frequentemente associadas a comprometimentos nas atividades cotidianas, na vida acadêmica e nas relações sociais ${ }^{12-14}$.

Os escolares com TDAH apresentam alterações na função executiva, que abrange todos os processos responsáveis por planejar, focalizar, guiar, direcionar e integrar as funções cognitivas, dentre elas o estado de alerta e as atenções sustentada e seletiva ${ }^{15-19}$. Devido a tais alterações, esses escolares apresentam dificuldade quanto ao aprendizado das habilidades metalinguísticas e aos aspectos fonológicos da linguagem, comprometendo aquisições posteriores, como a da leitura e da escrita, o que resulta em posteriores dificuldades de aprendizagem ${ }^{20,21}$.

A dislexia é um distúrbio específico de aprendizagem de origem neurológica, caracterizada pela dificuldade com a fluência correta na leitura e dificuldade na habilidade de deco- 
dificação e soletração, resultantes de um déficit fonológico da linguagem ${ }^{22}$. Os escolares com dislexia apresentam dificuldade na fluência correta da leitura, dificuldade na habilidade de decodificação, alteração na discriminação dos sons, consciência fonológica e limitação da memória de curta duração, podendo, também, ter problema com a memória verbal de longa duração, devido à dificuldade de formar léxico para a estocagem. Isso compromete seu desempenho na leitura de palavras irregulares, não frequentes, pseudopalavras, crescimento do vocabulário e na compreensão do material lido ${ }^{22-24}$.

Nos países que têm como língua oficial o espanhol, alguns pesquisadore ${ }^{25-30}$ vêm utilizando o PROLEC ${ }^{31}$ e o PROLEC - $\mathrm{SE}^{32}$ para avaliar e definir os processos de leitura em escolares do ensino fundamental e médio, respectivamente, procurando estabelecer um consenso entre os procedimentos utilizados para definir o perfil de escolares quanto ao desempenho em processos de leitura e seus preditores para a alfabetização ${ }^{30}$.

Mediante o exposto, o objetivo é caracterizar e comparar o desempenho de escolares com diagnóstico interdisciplinar de dislexia e TDAH com escolares com bom desempenho acadêmico nos processos de leitura.

\section{MÉTODO}

Este estudo foi realizado após aprovação do Comitê de Ética em Pesquisa da Faculdade de Filosofia e Ciências da Universidade Estadual Paulista - CEP/FFC/UNESP/ MaríliaSP, sob o protocolo de $\mathrm{n}^{\circ}$ 0579/2008.

Participaram deste estudo clínico com corte transversal 60 escolares na faixa etária de oito anos e dois meses a dez anos e onze meses de idade, de ambos os gêneros, de $1^{\mathrm{a}}$ a $4^{\mathrm{a}}$ série do ensino fundamental da cidade de Marília - SP, divididos em três grupos:
Grupo I (GI): composto por 20 escolares, 17 (85\%) do sexo masculino e três (15\%) do gênero feminino, com diagnóstico interdisciplinar de dislexia, sendo seis $(30 \%)$ da $2^{\mathrm{a}}$ série, sete $(35 \%)$ da $3^{\mathrm{a}}$ série e sete $(35 \%)$ da $4^{\mathrm{a}}$ série.

Grupo II (GII): composto por 20 escolares, $14(70 \%)$ do sexo masculino e $6(30 \%)$ do gênero feminino, com Transtorno do Déficit de Atenção e Hiperatividade.

Grupo III (GIII): composto por 20 escolares com bom desempenho acadêmico indicados por professores de escola pública do município de Marília, SP com base no desempenho satisfatório nas provas de Língua Portuguesa e Matemática em dois primeiros bimestres consecutivos, pareados segundo gênero, faixa etária e escolaridade com o GI.

O diagnóstico de dislexia foi realizado por equipe interdisciplinar do Laboratório de Investigação dos Desvios da Aprendizagem CEES/UNESP, de Marília, e do Ambulatório de Neurologia Infantil - Desvios da Aprendizagem da Faculdade de Medicina - FM/ UNESP, de Botucatu, incluindo avaliação fonoaudiológica, neurológica e neuropsicológica.

O diagnóstico de TDAH foi realizado por equipe interdisciplinar do Ambulatório de Neurologia Infantil - Desvios da Aprendizagem do Hospital das Clínicas da Faculdade de Medicina - HC/FM/UNESP - de Botucatu, incluindo avaliação fonoaudiológica, neurológica, neuropsicológica e seguindo critérios propostos pelo DSM-IV 9 .

A classificação do nível socioeconômico foi realizada com base no estudo estatístico do Índice de Desenvolvimento Socioeconômico - IDESE ${ }^{33}$, garantindo, dessa maneira, a homogeneidade da amostra do ponto de vista socioeconômico.

Neste estudo, foram realizados os seguintes procedimentos:

a) Termo de consentimento pós- informado: Conforme resolução do Conselho Nacional de Saúde CNS 196/96, anterior ao iní- 
cio das avaliações, os pais ou responsáveis dos pacientes selecionados assinaram o termo de consentimento pós-informado para a autorização da realização do estudo.

b) Avaliação dos processos de leitura: Os escolares foram submetidos à aplicação da adaptação brasileira da Avaliação dos Processos de Leitura - PROLEC ${ }^{34}$. Essa avaliação é composta por quatro blocos distribuídos para a avaliação de quatro processos de leitura, como descritos a seguir:

- $1^{\circ}$ Processo: Identificação de letras; composto por duas provas destinadas a medir a capacidade dos escolares para identificar as letras e seus respectivos sons. A prova de Identificação de som e letras tem por objetivo verificar a capacidade de o escolar nomear as letras e o som que as representa. A prova de Igual e diferente no que se refere a palavras e pseudopalavras visa verificar a capacidade de o escolar identificar, discriminar e reconhecer palavras reais e inventadas como sendo igual/ diferente.

- $2^{o}$ Processo: Processos léxicos; composto por quatro provas com a finalidade de comprovar o funcionamento das duas rotas de reconhecimento de palavras e seus subprocessos. Na prova de Decisão lexical, o escolar deve reconhecer apenas palavras reais em uma lista de palavras reais e inventadas independentemente de ser capaz de lê-las ou não.

Nas provas de Leitura de palavras, Leitura de pseudopalavras e Leitura de palavras e pseudopalavras, o objetivo é comparar o desenvolvimento das rotas de reconhecimento de palavras, e o escolar deve realizar a leitura de palavras reais e palavras inventadas, sendo que na primeira prova foi medida a capacidade de o escolar ler palavras reais e, na segunda, a capacidade para ler palavras inventadas de diferentes complexidades silábicas, divididas em CCV, VC, CVC, CVV, CCVC e CVVC.

$\mathrm{Na}$ terceira prova, o objetivo é analisar o grau de desenvolvimento que o escolar alcançou com o uso de rotas fonológica e lexical para leitura. Para isso, foram utilizadas palavras e pseudopalavras pertencentes a seis categorias: palavras de alta frequência curtas, palavras de alta frequência longas, palavras de baixa frequência curtas, palavras de baixa frequência longas, pseudopalavras curtas e pseudopalavras longas.

- $3^{\circ}$ Processo: Processos sintáticos; composto por duas provas. Na prova de Estruturas gramaticais é verificada a capacidade do escolar de processar diferentes tipos de estruturas gramaticais e comprovar a dificuldade que se pode produzir ao utilizar diferentes estruturas sintáticas, a saber: voz ativa, voz passiva e complemento focado. Na prova de Sinais de pontuação é verificada a capacidade de o escolar utilizar sinais de pontuação em um pequeno texto.

- $4^{o}$ Processo: Processos semânticos; composto por duas provas. Na prova de Compreensão de orações, o objetivo é avaliar se o escolar é capaz de extrair o significado de orações simples. Na prova de Compreensão de textos, o objetivo é investigar se o escolar é capaz de extrair o significado e integrá-lo aos seus conhecimentos.

Os escolares do GI foram avaliados no Laboratório de Avaliação e Intervenção dos Desvios da Linguagem, Fluência e Aprendizagem do Departamento de Fonoaudiologia da Faculdade de Filosofia e Ciências - CEES/ FFC/UNESP-Marília-SP e no Ambulatório de Neurologia Infantil - Desvios da Aprendizagem da Faculdade de Medicina - FM/UNESP - de Botucatu-SP.

Os escolares do GII foram avaliados no Ambulatório de Neurologia Infantil - Desvios da Aprendizagem do Hospital das Clínicas da Faculdade de Medicina - HC/FM/UNESP Botucatu, após 30 minutos da administração do medicamento (metilfenidato), uma vez que na ausência da medicação não foi possível realizar a avaliação proposta neste estudo. Os escolares do GIII foram avaliados na escola em uma sala de aula fornecida pela coordenação, 
em horário pré-determinado pelo professor de cada aluno. Foram realizadas duas sessões para a avaliação, com duração de 30 minutos cada.

Para a realização da análise estatística, foi utilizado o programa SPSS (Statistical Package for Social Sciences) em sua versão 19.0, com nível de significância de 5\% $(0,050)$. Aplicou-se o Teste de Kruskal-Wallis, com o intuito de verificarmos possíveis diferenças entre os três grupos estudados, quando comparados concomitantemente, para as variáveis de interesse; o Teste de Mann-Whitney, ajustado pela Correção de Bonferroni, para identificarmos quais grupos diferem-se dos demais, quando comparados par a par e o Teste da Razão de Verossimilhança, com o intuito de verificarmos possíveis diferenças entre os três grupos, quando comparados concomitantemente, seguida da aplicação do mesmo teste (com a Correção de Bonferroni), para identificação dos grupos que se diferem entre si, quando comparados par a par quanto à classificação do Prolec. A significância estatística foi marcada por asterisco $(*)$.

\section{RESULTADOS}

As Tabelas 1 e 2 apresentam os resultados da comparação entre os escolares de GI (disléxicos), GII (TDAH) e GIII (bom desempenho acadêmico) nas provas do PROLEC. Com a aplicação do Teste de Kruskal-Wallis, na Tabela 1, observa-se diferença estatisticamente significante em todas as provas dos processos léxico e na prova som e letra do proces-

Tabela 1: Comparação do desempenho dos escolares do GI, GII e GIII quanto ao desempenho nas provas dos processos de identificação de letras e léxico.

\begin{tabular}{|c|c|c|c|c|c|c|c|c|}
\hline $\begin{array}{c}\text { Processos de } \\
\text { Leitura }\end{array}$ & Variável & Grupo & Média & $\begin{array}{c}\text { Desvio } \\
\text { padrão }\end{array}$ & Mínimo & Máximo & Mediana & Valor de $\mathbf{P}$ \\
\hline \multirow{6}{*}{ 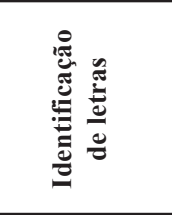 } & \multirow{3}{*}{ S_L } & GI & 17,35 & 3,31 & 8,00 & 20,00 & 18,00 & \multirow{3}{*}{$0,001 *$} \\
\hline & & GII & 18,00 & 3,45 & 9,00 & 20,00 & 19,50 & \\
\hline & & GIII & 19,95 & 0,22 & 19,00 & 20,00 & 20,00 & \\
\hline & \multirow{3}{*}{ ID } & GI & 18,85 & 1,18 & 16,00 & 20,00 & 19,00 & \multirow{3}{*}{0,121} \\
\hline & & GII & 18,25 & 4,46 & 0,00 & 20,00 & 20,00 & \\
\hline & & GIII & 19,60 & 0,50 & 19,00 & 20,00 & 20,00 & \\
\hline \multirow{21}{*}{ 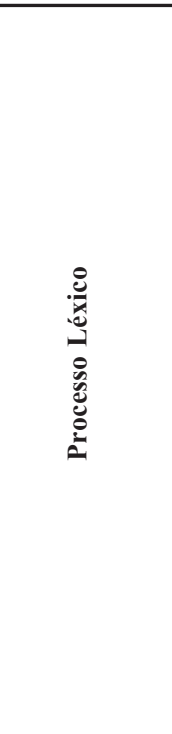 } & \multirow{3}{*}{ DL } & GI & 21,40 & 7,38 & 5,00 & 30,00 & 22,00 & \multirow{3}{*}{$0,023^{*}$} \\
\hline & & GII & 21,50 & 10,22 & 0,00 & 30,00 & 26,50 & \\
\hline & & GIII & 27,70 & 1,69 & 25,00 & 30,00 & 27,00 & \\
\hline & \multirow{3}{*}{ LP } & GI & 16,95 & 9,27 & 0,00 & 28,00 & 20,00 & \multirow{3}{*}{$<0,001^{*}$} \\
\hline & & GII & 22,00 & 10,65 & 0,00 & 30,00 & 27,00 & \\
\hline & & GIII & 29,25 & 1,21 & 26,00 & 30,00 & 30,00 & \\
\hline & \multirow{3}{*}{ LPP } & GI & 14,20 & 8,26 & 0,00 & 27,00 & 18,00 & \multirow{3}{*}{$<0,001^{*}$} \\
\hline & & GII & 19,75 & 10,18 & 0,00 & 29,00 & 24,00 & \\
\hline & & GIII & 27,20 & 2,26 & 22,00 & 30,00 & 28,00 & \\
\hline & \multirow{3}{*}{ LPF } & GI & 14,05 & 6,47 & 0,00 & 20,00 & 16,50 & \multirow{3}{*}{$<0,001^{*}$} \\
\hline & & GII & 15,25 & 7,42 & 0,00 & 20,00 & 19,00 & \\
\hline & & GIII & 19,90 & 0,31 & 19,00 & 20,00 & 20,00 & \\
\hline & \multirow{3}{*}{ LPNF } & GI & 11,45 & 5,04 & 0,00 & 17,00 & 13,00 & \multirow{3}{*}{$<0,001 *$} \\
\hline & & GII & 14,50 & 6,76 & 0,00 & 20,00 & 18,00 & \\
\hline & & GIII & 18,75 & 1,45 & 15,00 & 20,00 & 19,00 & \\
\hline & \multirow{6}{*}{ LNP } & GI & 9,85 & 4,91 & 0,00 & 15,00 & 12,00 & \multirow{6}{*}{$<0,001^{*}$} \\
\hline & & GII & 13,25 & 6,67 & 0,00 & 20,00 & 15,50 & \\
\hline & & GIII & 17,80 & 2,42 & 10,00 & 20,00 & 18,00 & \\
\hline & & GIII & 4,50 & 0,61 & 3,00 & 5,00 & 5,00 & \\
\hline & & GII & 7,30 & 5,07 & 0,00 & 13,00 & 9,50 & \\
\hline & & GIII & 13,55 & 2,11 & 9,00 & 16,00 & 14,00 & \\
\hline
\end{tabular}

Legenda: S L: som ou letra; ID: igual-diferente; DL: decisão léxica; LP: leitura de palavras; LPP: leitura de pseudopalavras; LPF: leitura de palavras frequentes; LPNF: leitura de palavras não freqüentes; LNP: leitura de não-palavras; ${ }^{*}$ estatisticamente significante. Teste de Kruskal-Wallis 
so de identificação de letras. Na tabela 2, observa-se diferença estatisticamente significante nas provas dos processos sintático e semântico, indicando que houve diferença no desempenho entre os três grupos de escolares. Observa-se que os escolares do GIII apresentaram desempenho superior em relação aos demais.

Tabela 2: Comparação do desempenho dos escolares do GI, GII e GIII quanto ao desempenho nas provas dos processos sintático e semântico

\begin{tabular}{|c|c|c|c|c|c|c|c|c|}
\hline $\begin{array}{l}\text { Processos de } \\
\text { Leitura }\end{array}$ & Variável & Grupo & Média & $\begin{array}{l}\text { Desvio } \\
\text { padrão }\end{array}$ & Mínimo & Máximo & Mediana & Valor de $P$ \\
\hline \multirow{6}{*}{ 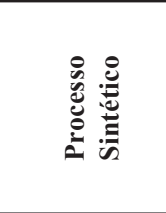 } & \multirow{3}{*}{ EG } & GI & 7,90 & 4,06 & 0,00 & 13,00 & 8,50 & \multirow{3}{*}{$0,001 *$} \\
\hline & & GII & 8,20 & 4,81 & 0,00 & 14,00 & 10,00 & \\
\hline & & GIII & 12,05 & 1,47 & 10,00 & 15,00 & 12,00 & \\
\hline & \multirow{3}{*}{ SP } & GI & 7,80 & 5,86 & 0,00 & 16,00 & 8,00 & \multirow{3}{*}{$<0,001 *$} \\
\hline & & GII & 5,95 & 4,20 & 0,00 & 10,00 & 8,00 & \\
\hline & & GIII & 13,15 & 1,60 & 10,00 & 16,00 & 13,00 & \\
\hline \multirow{6}{*}{ 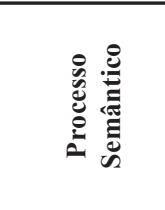 } & \multirow{3}{*}{$\mathrm{CO}$} & GI & 7,45 & 3,91 & 0,00 & 12,00 & 8,50 & \multirow{3}{*}{$<0,001^{*}$} \\
\hline & & GII & 7,45 & 4,43 & 0,00 & 12,00 & 9,50 & \\
\hline & & GIII & 11,85 & 0,37 & 11,00 & 12,00 & 12,00 & \\
\hline & \multirow{3}{*}{$\mathrm{CT}$} & GI & 5,65 & 4,04 & 0,00 & 12,00 & 6,00 & \multirow{3}{*}{$<0,001^{*}$} \\
\hline & & GII & 7,30 & 5,07 & 0,00 & 13,00 & 9,50 & \\
\hline & & GIII & 13,55 & 2,11 & 9,00 & 16,00 & 14,00 & \\
\hline
\end{tabular}

Legenda: EG: estrutura gramatical; SP: sinal de pontuação; CO: compreensão de orações; CT: compreensão de textos; * estatisticamente significante. Teste de Kruskal-Wallis

Como foram encontradas diferenças estatisticamente significantes para todas as comparações feitas nas Tabelas 1 e 2, foi aplicado o Teste de Mann-Whitney ajustado pela Correção de Bonferroni (Tabela 3), o que permitiu a identificação de quais grupos diferem dos demais a partir de uma comparação realizada par a par. Quando realizada a comparação entre os grupos GI e GII, foram encontradas diferenças estatisticamente significantes apenas nas provas de leitura de palavras não frequentes (LPNF) e leitura de não palavras (LNP) do processo léxico, o que evidencia desempenho superior dos escolares do GII em relação aos escolares do GI nessas provas e desempenho semelhante nas demais provas do PROLEC.

Tabela 3: Comparação do desempenho dos escolares do GI, GII e GIII nas provas dos processos de leitura, comparados par a par

\begin{tabular}{|c|c|c|c|c|}
\hline \multirow{2}{*}{$\begin{array}{l}\text { Processos } \\
\text { de Leitura } \\
\end{array}$} & \multirow[b]{2}{*}{ Variável } & \multicolumn{3}{|c|}{ Par de Grupos } \\
\hline & & GI X GII & GI X GIII & GII X GIII \\
\hline Identificação & S_L & 0,340 & $<0,001^{*}$ & $0,001^{*}$ \\
\hline de Letras & DL & 0,635 & $0,006^{*}$ & 0,055 \\
\hline \multirow{5}{*}{ Processo Léxico } & LP & 0,028 & $<0,001^{*}$ & $0,009^{*}$ \\
\hline & LPP & 0,038 & $<0,001^{*}$ & $0,008^{*}$ \\
\hline & LPF & 0,099 & $<0,001^{*}$ & $<0,001 *$ \\
\hline & LPNF & $0,005^{*}$ & $<0,001 *$ & $0,006^{*}$ \\
\hline & LNP & $0,010^{*}$ & $<0,001 *$ & $0,006^{*}$ \\
\hline Processo & EG & 0,568 & $<0,001^{*}$ & $0,004^{*}$ \\
\hline Sintático & SP & 0,279 & $0,005^{*}$ & $<0,001^{*}$ \\
\hline Processo & $\mathrm{CO}$ & 0,764 & $<0,001^{*}$ & $<0,001^{*}$ \\
\hline Semântico & $\mathrm{CT}$ & 0,194 & $<0,001^{*}$ & $<0,001 *$ \\
\hline
\end{tabular}

Teste de Mann-Whitney, ajustado pela Correção de Bonferroni (alfa de Bonferroni = 0,016952)

Legenda: S_L: som ou letra; ID: igual-diferente; DL: decisão léxica; LP: leitura de palavras; LPP: leitura de pseudopalavras; LPF: leitura de palavras frequentes; LPNF: leitura de palavras não freqüentes; LNP: leitura de não-palavras; EG: estrutura gramatical; SP: sinal de pontuação; CO: compreensão de orações; CT: compreensão de textos; *estatisticamente significante. 
Quando realizada a comparação entre os grupos GI e GIII, observa-se diferença estatisticamente significante em todas as provas do PROLEC, o que indica uma diferença significativa no desempenho de ambos os grupos. Já na comparação entre os grupos GII e GIII, observa-se diferença estatisticamente significante na maioria das provas, com exceção da provas de decisão lexical (DL) do processo léxico.
A Tabela 4 apresenta a comparação entre os escolares de GI, GII e GIII quanto à classificação das provas do PROLEC, e observase que dentre os escolares do GI a maioria apresentou classificação normal $(\mathrm{N})$ nas provas do processo de identificação de letras e na prova de sinais de pontuação do processo sintático, além de classificação dificuldade grande (DD) em todas as provas do processo léxi-

Tabela 4: Comparação da classificação normal (N), dificuldade pequena (D) e dificuldade grande (DD) nas provas dos processos de identificação de letras, léxico, sintático e semântico do PROLEC, entre os escolares dos grupos GI, GII e GIII

\begin{tabular}{|c|c|c|c|c|c|c|c|c|}
\hline Processos de Leitura & Classificação & GI & GII & GIII & I x II x III & I x II & I x III & II x III \\
\hline \multirow{6}{*}{ 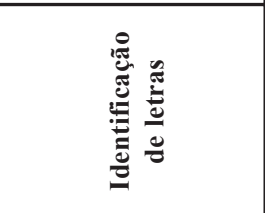 } & S.L. N & 9 & 14 & 20 & \multirow{3}{*}{$0,004^{*}$} & \multirow{3}{*}{0,249} & \multirow{3}{*}{$0,001^{*}$} & \multirow{3}{*}{0,029} \\
\hline & S.L.D. & 3 & 1 & 0 & & & & \\
\hline & S.L.DD. & 8 & 5 & 0 & & & & \\
\hline & ID.N & 14 & 16 & 20 & \multirow{3}{*}{$0,003 *$} & \multirow{3}{*}{0,031} & \multirow{3}{*}{0,029} & \multirow{3}{*}{0,108} \\
\hline & ID.D. & 5 & 0 & 0 & & & & \\
\hline & ID.DD. & 1 & 4 & 0 & & & & \\
\hline \multirow{18}{*}{ 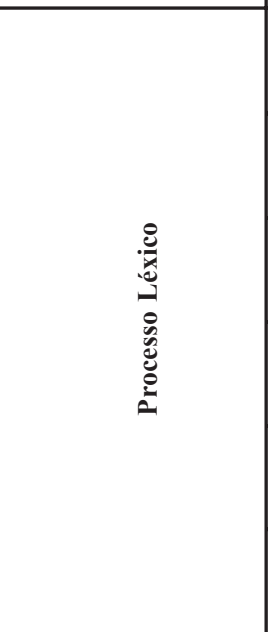 } & DL.N. & 8 & 12 & 18 & \multirow{3}{*}{$0,004 *$} & \multirow{3}{*}{0,272} & \multirow{3}{*}{$0,001 *$} & \multirow{3}{*}{0,027} \\
\hline & DL.D. & 1 & 2 & 2 & & & & \\
\hline & DL.DD. & 11 & 6 & 0 & & & & \\
\hline & LP.N & 0 & 10 & 19 & \multirow{3}{*}{$<0,001^{*}$} & \multirow{3}{*}{$0,001^{*}$} & \multirow{3}{*}{$<0,001 *$} & \multirow{3}{*}{$0,006^{*}$} \\
\hline & LP.D. & 3 & 2 & 0 & & & & \\
\hline & LP.DD & 17 & 8 & 1 & & & & \\
\hline & LPP.N & 1 & 9 & 19 & \multirow{3}{*}{$<0,001^{*}$} & \multirow{3}{*}{$0,014^{*}$} & \multirow{3}{*}{$<0,001 *$} & \multirow{3}{*}{$0,003 *$} \\
\hline & LPP.D & 2 & 1 & 0 & & & & \\
\hline & LPP.DD & 17 & 10 & 1 & & & & \\
\hline & LPF.N & 5 & 13 & 20 & \multirow{3}{*}{$<0,001^{*}$} & \multirow{3}{*}{$0,015^{*}$} & \multirow{3}{*}{$<0,001 *$} & \multirow{3}{*}{$0,014 *$} \\
\hline & LPF.D & 4 & 0 & 0 & & & & \\
\hline & LPF.DD & 11 & 7 & 0 & & & & \\
\hline & LPNF.N & 0 & 12 & 19 & & & & \\
\hline & LPNF.D & 5 & 1 & 0 & $<0,001^{*}$ & $<0,001 *$ & $<0,001 *$ & 0,029 \\
\hline & LPNF.DD & 15 & 7 & 1 & & & & \\
\hline & LNP.N & 0 & 7 & 16 & & & & \\
\hline & LNP.D & 0 & 4 & 2 & $<0,001 *$ & $0,001^{*}$ & $<0,001^{*}$ & $0,013^{*}$ \\
\hline & LNP.DD & 20 & 9 & 2 & & & & \\
\hline & EG.N & 7 & 11 & 20 & & & & \\
\hline & EG.D & 3 & 2 & 0 & $0,001 *$ & 0,356 & $<0,001^{*}$ & $0,005^{*}$ \\
\hline$\tilde{z}=\stackrel{\tilde{s}}{=}$ & EG.DD & 10 & 6 & 0 & & & & \\
\hline 巳 & SP.N & 15 & 13 & 20 & & & & \\
\hline$\therefore$ i & SP.D & 0 & 0 & 0 & 0,087 & - & - & - \\
\hline & SP.DD & 5 & 7 & 0 & & & & \\
\hline & CO.N & 5 & 7 & 20 & & & & \\
\hline & CO.D & 1 & 2 & 0 & $<0,001 *$ & 0,598 & $<0,001 *$ & $<0,001^{*}$ \\
\hline $\bar{z}$ & CO.DD & 14 & 11 & 0 & & & & \\
\hline 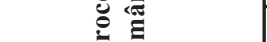 & CT.N & 3 & 4 & 17 & & & & \\
\hline 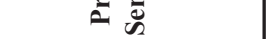 & CT.D & 3 & 5 & 2 & $<0,001^{*}$ & 0,606 & $<0,001 *$ & $<0,001 *$ \\
\hline & CT.DD & 14 & 11 & 1 & & & & \\
\hline
\end{tabular}

Teste da Razão de Verossimilhança, com a Correção de Bonferroni

Legenda: S_L: som ou letra; ID: igual-diferente; DL: decisão léxica; LP: leitura de palavras; LPP: leitura de pseudopalavras; LPF: leitura de palavras frequentes; LPNF: leitura de palavras não freqüentes; LNP: leitura de não-palavras; EG: estrutura gramatical; SP: sinal de pontuação; CO: compreensão de orações; CT: compreensão de textos; *estatisticamente significante. 
co, do processo semântico e na prova estrutura gramatical (EG) do processo sintático.

Quanto ao GII, a maioria dos escolares apresentou classificação dificuldade grande(DD) em todas as provas do processo semântico e na leitura de pseudopalavras do processo léxico e classificação normal $(\mathrm{N})$ nas demais provas desse processo, nas provas de identificação de letras e nas do processo sintático. Já o GIII apresentou a maioria dos escolares com classificação normal $(\mathrm{N})$ em todas as provas do PROLEC.

Quando realizada a comparação entre os grupos par a par, com a aplicação do Teste da Razão de Verossimilhança com a correção de Bonferroni, observa-se que em GI x GII houve diferença estatisticamente significante em cinco provas, das seis, do processo léxico (exceto em decisão lexical), e não houve diferença nos demais processos. Isso evidencia desempenho similar do GI e do GII nas provas do PROLEC.

Na comparação GI x GIII houve diferença estatisticamente significante em todas as provas dos processos léxicos e semânticos, na prova som-letra (SL) do processo identificação de letras e na prova estrutura gramatical (EG) do processo semântico, o que indica desempenho superior de GIII, comparado a GI, nas provas do PROLEC.

Ao comparar GII x GIII, observa-se diferença estatisticamente significante na maioria das provas do processo léxico (exceto decisão lexical - DL e leitura de palavras não frequentes - LPNF), na prova estrutura gramatical do processo sintático e em todas as provas do processo semântico. Isso evidencia desempenho superior do GIII, comparado ao GII, nas provas do PROLEC.

\section{DISCUSSÃO}

A partir dos achados deste estudo, observa-se que ao comparar o desempenho dos escolares do GI e do GII com os escolares do GIII, verificou-se diferença estatisticamente significante em todas as provas, evidenciando um desempenho superior de GIII em relação aos demais escolares. Esse dado pode ser justificado pelo fato de que consciência fonológica, memória e acesso a informação fonológica estocada na memória de longa duração são importantes para o desenvolvimento da leitura, e alterações nessa habilidade são geralmente identificadas em escolares com problemas na aprendizagem ${ }^{25,26,28,30,35-37}$.

Quando realizada a comparação entre os escolares do GI e do GII, observa-se diferença estatisticamente significante apenas nas provas de leitura de palavras não frequentes (LPNF) e leitura de não palavras (LNP) do processo léxico, corroborando a literatura que afirma que a habilidade de codificação, alteração em consciência fonológica, limitação da memória e na formação de léxico prejudicam o desenvolvimento das habilidades necessárias para a leitura ${ }^{23,24,38-40}$, características em comum encontradas na dislexia e no TDAH.

Quanto à classificação das provas do PROLEC, observa-se que dentre os escolares do GI a maioria apresentou classificação normal $(\mathrm{N})$ nas provas do processo de identificação de letras e na prova de sinais de pontuação do processo sintático, além de classificação dificuldade grande (DD) em todas as provas do processo léxico, do processo semântico e na prova estrutura gramatical (EG) do processo sintático. Esse resultado corrobora estudos que afirmam que disléxicos apresentam dificuldades na conversão grafema-fonema, prejudicando o desenvolvimento da leitura, a discriminação de sons e a memória verbal ${ }^{24,37,41}$.

Tais achados corroboram, ainda, estudos espanhóis que utilizaram o PROLEC ${ }^{31}$ para avaliar a leitura em disléxicos, pois verificaram dificuldades em provas que exigiram a habilidade fonológica ${ }^{25,26,28,30}$.

Quanto ao GII, a maioria dos escolares apresentou classificação dificuldade grande (DD) em todas as provas do processo semântico e nas provas de leitura de não palavras (LNP) 
e leitura de pseudopalavras (LPP) do processo léxico, e classificação normal $(\mathrm{N})$ nas demais provas do processo léxico, nas provas de identificação de letras e nas do processo sintático. Tais achados justificam-se pelo comprometimento da interação entre processamento visual, linguístico e auditivo encontrado em escolares com TDAH. Esse comprometimento resulta em falhas na decodificação fonológicas, as quais geralmente impedem o acesso ao significado das palavras e dos textos, o que compromete a compreensão do texto lido ${ }^{21,39,40,42,43}$.

Diante do exposto, este estudo evidenciou que a dificuldade apresentada pelo GI e pelo GII nas provas do PROLEC pode ser justificada pela dificuldade de codificação e decodificação de símbolos. Nos escolares com TDAH, essa alteração se deve ao comprometimento da interação entre o processamentos visual, linguístico, atencional e auditivo, e nos escolares com dislexia é em decorrência de falha no processo de mediação fonológica, que depende da utilização do conhecimento das regras de conversão grafema-fonema para a construção da leitura da palavra. Essas alterações comprometem a realização da leitura e a compreensão do material lido.

\section{REFERÊNCIAS}

1. Harn BA, Stoolmiller M, Chard, DJ. Measuring the Dimensions of Alphabetic Principle on the Reading Development of First Graders. The Role of Automaticity and Unitization. J Learn Disabil. 2008; 41(2): 143-57.

2. Cunha VLO, Capellini, SA. . Desempenho de escolares de $1^{\mathrm{a}}$ a $4^{\mathrm{a}}$ série do ensino fundamental nas provas de habilidades metafonológicas e de leitura - PROHMELE. Rev. Soc Bras Fonoaudiol. 2009; 14(1): 56-68.
Assim, os achados deste estudo permitem concluir que os escolares de GIII com bom desempenho acadêmico apresentaram desempenho superior nas provas do PROLEC em relação aos escolares de GI e GII. A dificuldade apresentada pelos GI e GII nas provas do PROLEC pode ser justificada pela dificuldade de codificação e decodificação de símbolos. Nos escolares com TDAH, essa alteração se deve ao comprometimento da interação entre os processamentos visual, linguístico, atencional e auditivo e, nos escolares com dislexia, é em decorrência de falha no processo de mediação fonológica, que depende da utilização do conhecimento das regras de conversão grafema-fonema para a construção da leitura da palavra. Tais alterações comprometem a realização da leitura e a compreensão do material lido.

É necessária a continuidade de estudos com um maior número de escolares para melhor definir se o perfil de leitura encontrado neste estudo permanece o mesmo, favorecendo, assim, maior informação clínica e educacional para a diferenciação dos diagnósticos e no planejamento de intervenções clínicas e educacionais.

3. Barrera SD, Maluf MR. Consciência metalinguística e alfabetização: um estudo com crianças da primeira série do ensino fundamental. Psicol Reflex Crit. 2003;16(3):491-502.

4. Gindri G, Keske-Soares M, Mota HB. Memória de trabalho, consciência fonológica e hipótese de escrita. Pró-Fono: Rev Atual Cient. 2007;19(3):313-22.

5. American Academy of Pediatrics. Clinical practice guideline: diagnosis and evaluation of the child with attentiondefict/hyperactivity disorder. Pediatrics. 2000; 105: 1158-1170. 
6. Barkley RA, Fischer M, Smallish L, Fletcher K. The persistence of attentiondeficit/hyperactivity disorder into young adulthood as a function of reporting source and definition of disorder. J Abnorm Child Psychol.2002;111(2):279-89.

7. Faraone SV. Report from the $4^{\text {th }}$ international meeting of the attention deficit hyperactivity disorder molecular genetics network. Am J Med Genet. 2003; 121(1): 55-9.

8. Mattos P, Palmini A, Salgado CA, Segenreich D, Grevet E, Oliveira IR et al. Painel brasileiro de especialistas sobre diagnóstico do transtorno de déficit de atenção/hiperatividade (TDAH) em adultos. Rev Psiquiatr RS.2006;28(1):50-60.

9. American Psychiatric Association. Diagnostic and Statistical Manual of Mental Disorders. (DSM-IV) 4a ed. Washington (DC): American Psychiatric Association; 1994. p.46-53.

10. Graeff RL, Vaz CE. Personalidade de Crianças com transtorno de déficit de atenção e hiperatividade (TDAH) por meio do Rorschach. Psic Teor Pesq.2006; 22(3):269-76.

11. Rohde LA, Biederman J, Busnello EA, Zimmermann H, Schmitz M, Martins S et al. ADHD in a school sample of Brazilian adolescents: a study of prevalence, comorbid conditions, and impairments. $\mathrm{J}$ Am Acad Cild Adolesc Psychiatry. 1999; 38: 716-22.

12. Kessler RC, Adler LA, Barkley R, Conner CK, Faraone SV, Greenhill LL, Jaeger S et al. Patterns and Predictor of attentiondeficit/hyperactivity disorder persistence into adulthood: Results from the national comorbidity survey replication. Biol Psychiatry.2005;57(11):1442-51.

13. Lima CC, Albuquerque G. Avaliação de linguagem e co-morbidade com transtornos de linguagem. In: Rohde LA, Mattos
P, editors. Princípios e práticas em TDAH. Porto Alegre: Artmed; 2003. p.117-42.

14. Pastura GMC, Mattos P, Araújo APQC. Desempenho escolar e transtorno do déficit de atenção e hiperatividade. Revista de Psiquiatria Clínica. 2005;32(6):324-9.

15. Capovilla AGS, AssefECS, Cozza HFP. Avaliação neuropsicológica das funções executivas e relação com desatenção e hiperatividade. Aval Psicol. 2007;6(1):51-60.

16. Capovilla AGS, Cozza HFZ, Capovilla FC, Macedo EC. Funções executivas em crianças e correlação com desatenção e hiperatividade. Temas Desenvolv. 2005; 14(82): 4-14.

17. Grevet EH, Abreu PB, Shansis F. Proposta de uma abordagem psicoeducacional em grupos para pacientes com transtorno de déficit de atenção/hiperatividade. Rev Psiquiatr RS.2003;25(3):446-52.

18. Hurks PPM, Hendriksen JGM, Vles JSH, Kalff AC, Feron FJM, Kroes M. et al. Verbal fluency over time as a measure of automatic and controlled processing in children with ADHD. Brain and Cognition. 2004;55(3):535-44.

19. Saboya E, Saraiva D, Palmini A, Lima P, Coutinho G. Disfunção executiva como uma medida de funcionalidade em adultos com TDAH. J Bras Psiquiatr. 2007; 56(Supl1): 30-3.

20. Ygual-Fernández A, Miranda-Casas A, Cervera-Mérida JF. Dificultades em las dimensiones de forma y contenido del lenguaje em los niños com trastorno por déficit de atención com hiperactividad. Rev Neurol Clín.2000;1:193-202.

21. Gallardo-Paúls B, Martinéz MG, Campos VM. Pragmática textual em adolescentes con trastorno por déficit de atención/ hiperactividad: argumentación. Rev Neurol. 2010;50(Sup13):113-7. 
22. Lyon GR, Shaywitz SE, Shaywitz BA. Defining dyslexia, comorbidity, teacher's knowledge of language and reading. Ann Dyslexia. 2003,53:1-14.

23. Germano GD, Capellini SA. Eficácia do programa de remediação auditivo-visual computadorizado em escolares com dislexia. Pró-Fono: Rev Atual Cient. 2008; 20(4): 237-42.

24. Pennala R, Eklund $\mathrm{K}$, Hämäläinen $\mathrm{K}$, Richardson U, Martin M, Leiwo M, et

al. Perception of phonemic length and its relation to reading and spelling skills in children with family risk for dyslexia in the first three grades of school. J Speech Lang Hear Res, 2010 Jun; 53 (3):710-724.

25. Escribano CL. Evaluation of the doubedeficit hypothesis subtype classification of reader in Spanish. J Learn Disabil. 2007; 40(4): 319-30.

26. Bedmarek D, Saldaña D, García I. Visual versus phonological abilities in Spanish dyslexic boys and girls. Brain and Cognition. 2009;70:273-8.

27. Cerdán R, Abarca EV, Martínez T, Gilabert $\mathrm{R}$, Gil L. Impacto f question-answering tasks on research processes and reading comprehension. Learning and Instruction.2009;19:13-27.

28. Jiménez JE, Rodríguez C, Ramírez G. Spanish developmental dyslexia. Prevalence, cognitive profile, and home literacy experiences. J Exp Child Psychol. 2009; 103:167-85.

29. Ponsa I, Quiroga JAR, Ribasés M, Bosch R, Bielsa A, Ordeig MT, Morell M, Miró $\mathrm{R}$, Cid R, et al. Absence of cytogenetic effects in children and adults with attention-deficit/hyperactivity disorder treated with methylphenidate. Mutation Research.2009;666:44-9.

30. Villagrán MA, Guzmán, JIN, Jiménez IM, Cuevas CA, Consejeto, EM, Olivier PR.
Velocidad de nombrar y conciencia fonológica en el aprendizaje inicial de la lectura. Psicothema.2010;22(3):436-42.

31. Cuetos F, Rodríguez B, Ruano E. Evaluación de los procesos lectores. Madrid: TEA Ediciones; 1996.

32. Ramos JL, Cuetos F. PROLEC-SE, Evaluación de los procesos lectores em alumnos de 3er ciclo de primaria y secundaria (b). Madrid: TEA Ediciones; 1999.

33. Secretaria da Coordenação e Planejamento, Fundação de Economia e Estatística (FEE). Índice de desenvolvimento Socioeconômico (IDESE). 2005 [acesso em 20 jan 2011] Disponível em: http:// www.fee.tche.br/sitefee/pt/content/ estatisticas/pg_idese.php

34. Capellini SA, Oliveira AM, Cuetos F. PROLEC: Provas de avaliação dos processos de leitura. São Paulo (SP): Casa do Psicólogo; 2010.

35. Piasta SB, Wagner RK. Learning letter names and sounds: Effects of instruction, letter type, and phonological processing skill. J Exp Child Psychol. 2010; 105(4): 324-344.

36. Germano GD, Pinheiro FH, Cunha VLO. Avaliação e intervenção nas habilidades metalinguísticas In: Capellini SA, Germano GD, Cunha VLO. Transtornos de aprendizagem e transtornos da atenção: da avaliação à intervenção. São José dos Campos, SP: Pulso Editorial. 2010. p.35-48.

37. Pinheiro FH, Germano GD, Capellini SA. A influência do treinamento auditivo para o desenvolvimento de habilidades fonológicas e silábicas em escolares com distúrbio de aprendizagem. Temas desenvolvimento. 2010;17:79-84.

38. Messina LF, Tiedemann, KB. Avaliação da memória de trabalho em crianças com transtorno do déficit de atenção e hiperatividade. Psicol USP.2009,20(2):209-28. 
39. Oliveira CG; Albuquerque PB. Diversidade de resultados no estudo do transtorno de déficit de atenção e hiperatividade. Psic.: Teor. e Pesq.2009;25(1):93-102.

40. Miranda-Casas A, Fernandéz MI, Robledo P, García-Castellar R. Comprensión de textos de estudiantes con trastorno por déficit de atención/hiperactividad: ¿qué papel desempeñan las funciones ejecutivas? Rev Neurol. 2010; 50(supl3):135-42.

41. O'Connor RE, Swanson HL, Geraghty C. Improvement in reading rate under independent and difficult text levels: Influences on word and comprehension skills. J Educ Psychol. 2010;102(1):1-19.
42. Lobo, PAS, Lima, LAM. Comparação do desempenho em leitura de palabras de crianças com e sem transtorno de déficit de atenção/hiperatividade. Rev Cefac 2008; 10(4):471-83.

43. Papazian O, Alfonso I, Luzondo RJ, Araguez N. Entrenamiento de la función ejecutiva en pré escolares con trastorno por déficit de atención/ hiperactividad combinado: estudio prospectivo, controlado y aleatorizado. Rev. Neurol. 2009; 48 (supl2): 119-22.

Recebido em: 08/ago./2010

Modificado em 26/nov./2010

Aceito em 16/mar./2011 